\title{
A Black Hole in a Bone - Intraosseous Lipoma
}

PETER MANDL, MD, PhD; ADAM MESTER, MD, PhD; PETER V. BALINT, MD, PhD, FRCP, National Institute of Rheumatology and Physiotherapy, 25-29 Frankel Leo str., H-1023, Budapest, Hungary. Address reprint requests to Dr. Mandl; E-mail: mandlpeter@yahoo.com. J Rheumatol 2009;36:466-8; doi:10.3899/jrheum.080731

Intraosseous lipoma is an extremely rarely diagnosed benign bone tumor. In most cases diagnostic imaging techniques, namely computed tomography (CT) and magnetic resonance imaging (MRI) are sufficient to reach a diagnosis, which in some cases is confirmed by postsurgical or postmortem histological examination. We describe 2 cases of intraosseous lipoma that were identified by practising rheumatologists at our rheumatology clinic.

A 38-year-old man presented with pain in his right foot, ankle, knee, and first metatarsophalangeal joint. His history featured trauma-induced fractures of the left ankle and the right 6th rib. He did not recall any recent infection, travel, exertion, or other illness. Examination including musculoskeletal examination yielded no significant findings.
Laboratory tests including metabolic panel and parathyroid hormone values were normal. Conventional radiographs of the right ankle and foot revealed a round, translucent, cystic lesion in the right calcaneal diaphysis in the trabecular bone, with areas of calcification (Figure 1a). CT scan of the lesion showed complete resorption of bone within the lesion, with the remaining tissue showing a fat attenuation level (-77 Hounsfield units; Figure 1b). The presence of fat within the lesion ruled out the diagnosis of intraosseous gouty tophus. On T1-weighted MRI scans the well delineated lesion appeared to be isointense with subcutaneous fat (Figure 1c), while significantly lower signal intensity was observable on T2-weighted images (Figure 1d).

The second patient, a 61-year-old man, had a history of

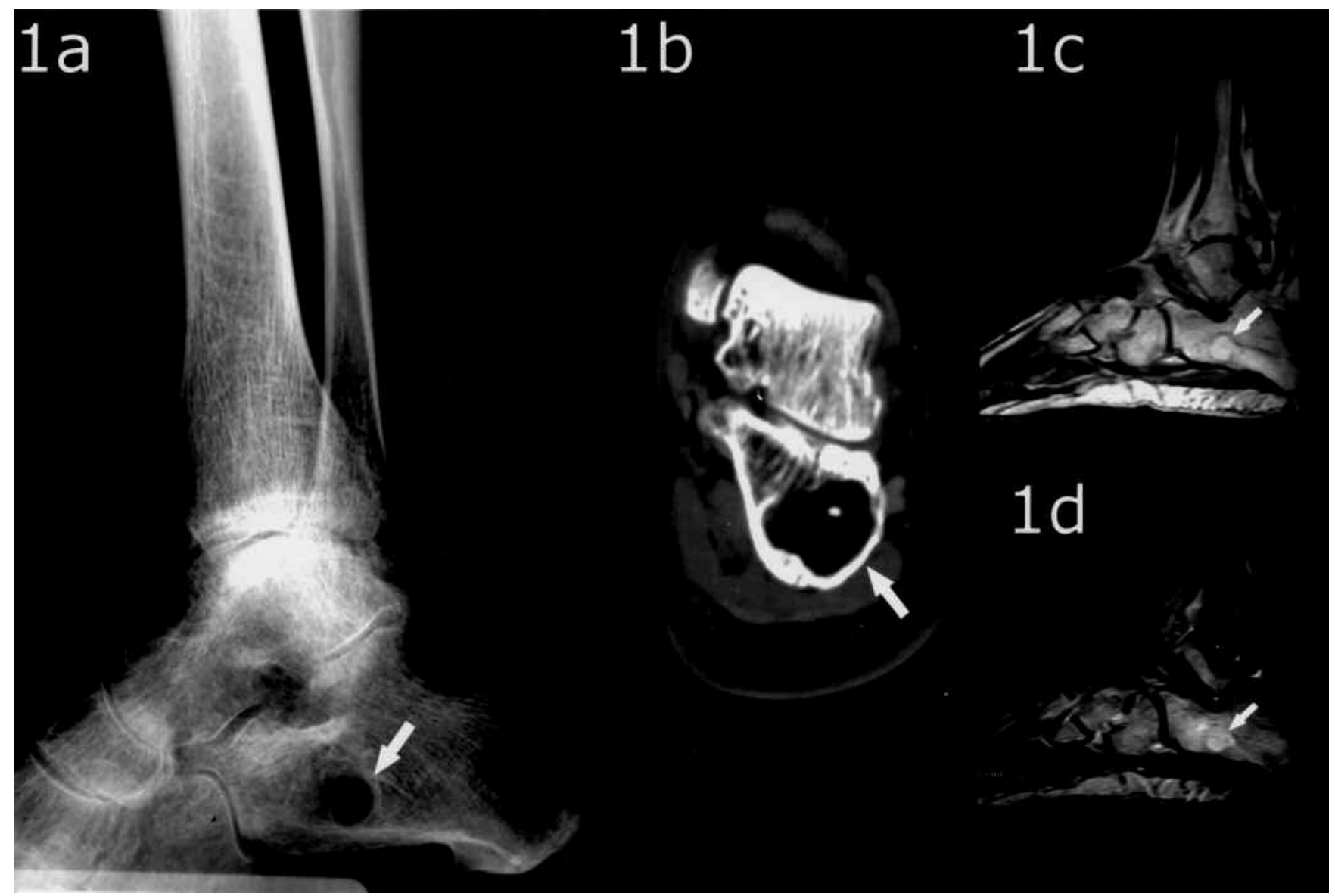

Figure 1. a. Plain radiograph. b. CT scan. c. T1-weighted MRI scan. d. T2-weighted MRI scan of the right ankle. Arrows show the lesion.

Personal non-commercial use only. The Journal of Rheumatology Copyright @ 2009 . All rights reserved. 
sport-related injuries culminating in meniscectomies carried out on both knees. He also suffered a trimalleolar fracture of the right ankle in 2002, but presented at our clinic in 2003 with pain in the left hip. He reported slight difficulty walking. Examination including musculoskeletal examination yielded no abnormality, but the anteroposterior radiograph of the pelvis revealed a round lesion in the other femoral head (diameter $30 \mathrm{~mm}$; Figure 2a), where he had not complained of symptoms. Laboratory tests including metabolic panel and parathyroid hormone values were normal. The lesion resembled a "black hole" when viewed under CT (Figure 2b), with a main Hounsfield unit of -63 that corresponded to adipose tissue. The lesion appeared to be isointense with subcutaneous fat on T1-weighted MRI scans and exhibited low signal intensity with fat suppression on T2weighted images (Figure 2c, 2d).

Intraosseous lipomas are rare benign neoplasms that represent proliferation of fat tissue within the marrow of normal trabecular bone ${ }^{1}$. Intraosseous lipomas occur primarily in the long bones of the lower extremities (i.e., femur, tibia, calcaneus), which are characterized by a scarcity of trabecular bone. They can also occur in the flat bones, pelvis, and other locations ${ }^{2,3}$. Milgram classified intraosseous lipomas according to the degree of involution (necrosis, calcification, cyst formation, and reactive ossification) observable in the lesion. Based on this classification intraosseous lipomas were categorized into 3 types - Stage 1 tumors consisting of viable fat cells; Stage 2 tumors composed partly of viable fat cells also demonstrating fat necrosis and calcification; and Stage 3 lesions with full range of involution: necrotic fat, calcification of necrotic fat, variable degrees of cyst formation, and reactive woven bone formation ${ }^{4}$. Typically, plain radiographs suggest the diagnosis, which is subsequently confirmed by CT or MRI with fat-suppression sequences $^{5}$.
In both of our cases plain radiographs showed round, translucent, cystic lesions with sclerotic margins. A classical appearance of intraosseous lipoma of the calcaneus is the presence of central calcification within a well defined lytic lesion resembling a cockade. The calcaneal lesion seen in Patient 1 also showed similar areas of increased radiographic density due to fat calcification. CT scans showed complete resorption of bone within the lesions, with the remaining tissue showing a fat attenuation level (-77 and -63 Hounsfield units, respectively). A hypointense rim was observable surrounding both lesions, consistent with reactive sclerosis. Both lesions appeared to be isointense, with subcutaneous fat on T1-weighted MRI scans exhibiting low signal intensity with fat suppression on T2-weighted images. The 2 cases correspond to stage 2 and stage 1 intraosseous lipomas, respectively, according to Milgram's classification.

Modern imaging techniques have proven to be reliable in the diagnosis of intraosseous lipoma ${ }^{6}$. In some cases fatty involution of an aneurysmal bone cyst, or bone infarct, may not be ruled out unequivocally ${ }^{7}$; malignant tumors and the malignant transformation of benign lesions should also be considered $^{8}$. In such cases a guided biopsy is required for the correct diagnosis. Complete removal is indicated in cases leading to potential pathological fractures. In the majority of cases lipomas run an indolent course, and can be followed by a wait and scan approach ${ }^{9}$. Due to the unequivocal diagnosis based on imaging modalities and lack of clinical progression over a course of more than 5 years, our 2 cases did not require biopsy or surgery, with followup consisting of regular scans.

\section{REFERENCES}

1. Lanisnik B, Didanovic V. Sphenoclival intraosseus lipoma: case report and literature review. Skull Base 2007;17:211-4.

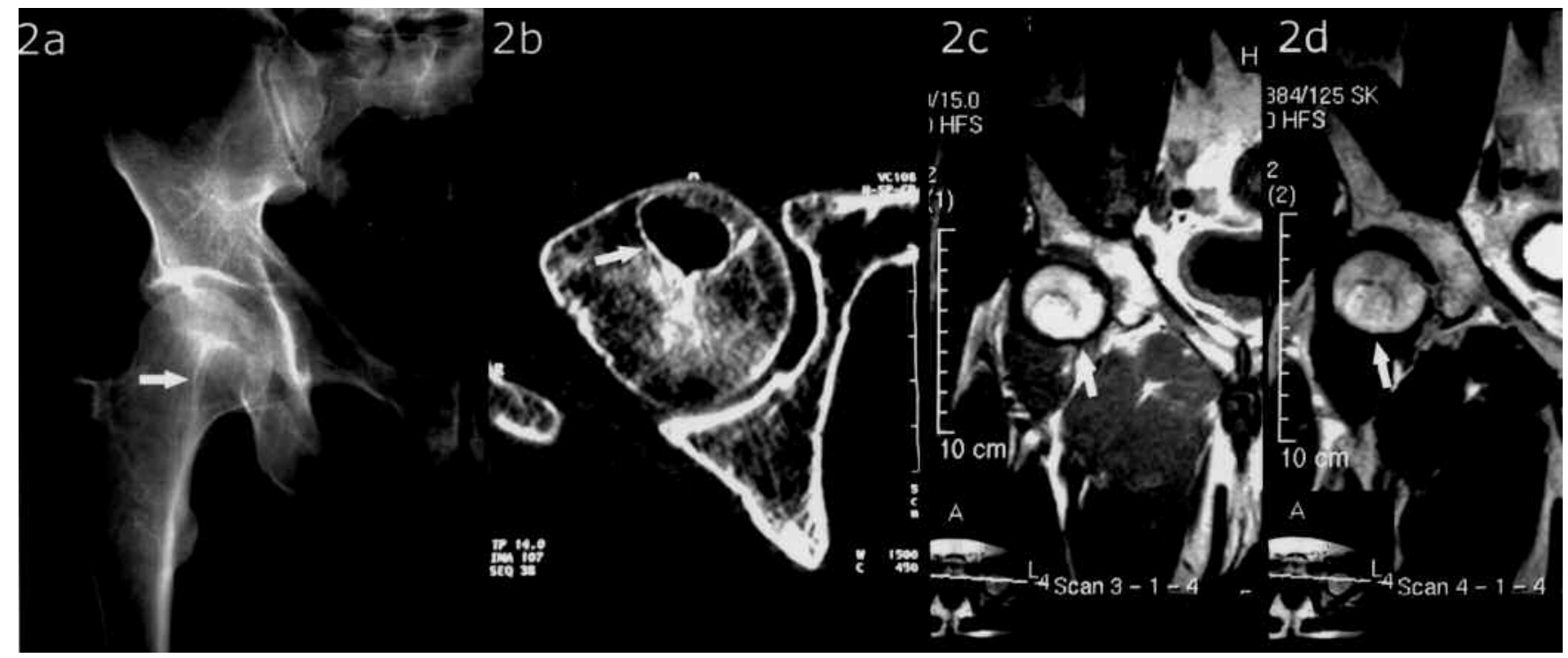

Figure 2. a. Plain radiograph. b. CT scan. c. T1-weighted MRI scan. d. T2-weighted MRI scan of the right femoral head. Arrows show the lesion.

Personal non-commercial use only. The Journal of Rheumatology Copyright (c) 2009. All rights reserved. 
2. MacFarlane MR, Soule SS, Hunt PJ. Intraosseous lipoma of the body of the sphenoid bone. Clin Neurosci 2005;12:105-8

3. Solak O, Esme H, Sahin DA, Aktepe F. Giant intraosseous lipoma of the rib. Thorac Cardiovasc Surg 2007;55:273-4.

4. Milgram JW. Intraosseous lipomas: radiologic and pathologic manifestations. Radiology 1988;167:155-60.

5. Blacksin MF, Ende N, Benevenia J. Magnetic resonance imaging of intraosseous lipomas: a radiologic-pathologic correlation. Skeletal Radiol 1995;24:37-41.
6. Coquerelle P, Cotten A, Flipo RM, Chastanet P, Duquesnoy B, Delcambre B. Intraosseous lipoma: role and limitations of modern imaging techniques. Rev Rhum Engl Ed 1988;62:147-50.

7. El-Atta MA, Ivancevich SM, Braunstein EM. Intraosseous lipoma. Arthritis Rheum 1997;40:978-9.

8. Kyriakos M, Hardy D. Malignant transformation of aneurysmal bone cyst, with an analysis of the literature. Cancer 1991;68:1770-80.

9. Hirata M, Kusuzaki K, Hirasawa Y. Eleven cases of intraosseous lipoma of the calcaneus. Anticancer Res 2001;21:4099-103. 